\title{
REFINEMENTS OF A CLASSICAL TECHNIQUE OF AIRGLOW SPECTROSCOPY
}

\author{
J. Scheer and E. R. Reisin ${ }^{1}$ \\ ${ }^{1}$ Instituto de Astronomía y Física del Espacio, CONICET, Ciudad Universitaria, 1428 Buenos Aires, \\ Argentina
}

\begin{abstract}
We have performed ground-based airglow observations with a tilting filter spectrometer to determine rotational temperatures in the mesopause region since 1984. Although quite simple in principle, the success of the technique depends on a number of non-trivial details, including corrections for measurements under less favorable conditions, as, for instance, full moon nights. Successive improvements have finally led to nearly continuous observations, since August 1997, with more than 200 nights of useful data per year. The technique is described in some detail and recent results are discussed.
\end{abstract}

\section{INTRODUCTION}

The general advantages of interference filter photometers are exploited widely in modern instruments like scanning tilting filter spectrophotometers (e.g., Takahashi et al., 1998) and airglow imagers (e.g., Taylor et al. 1999) for studying upper atmosphere dynamics by measuring intensity and rotational temperature. Those advantages are high sensitivity (due to large throughput and moderate bandwidth), and intrinsically high short- and long-term stability. Also our zenith-looking tilting filter spectrometer in use in Argentina since 1984 (Scheer, 1987) has these benefits. A series of hardware and software features and analysis refinements have contributed to prevent this relatively simple instrument from becoming obsolete. The purpose of this paper is to review these details, of the kind which are often not dealt with in the literature, because we think they may be useful also for new instrument developments.

\section{INSTRUMENT}

Our tilting filter spectrometer measures rotational temperatures of the $\mathrm{OH}(6-2)$ and the $\mathrm{O}_{2}(0-1)$ airglow bands (in the same sampling sequence and so interlaced that they correspond to the same effective time; see below), which correspond to altitudes of 87 and $95 \mathrm{~km}$, respectively. The instrument consists essentially of an interference filter (with $48 \mathrm{~mm}$ diameter and about $1.4 \mathrm{~nm}$ normal incidence bandwidth), a tilting mechanism moved by a stepping motor, and a GaAs photomultiplier (RCA C31034) cooled to $-35^{\circ} \mathrm{C}$ and operated in photon counting mode. Its wide spectral range from 844.5 to $867.2 \mathrm{~nm}$ is not covered by a linear scan. Instead, only seven spectral positions are sampled in rapid succession: three P-branch components ( $\mathrm{P} 4$ to $\mathrm{P} 6$ ) of the $\mathrm{OH}$ band, a background position between the $\mathrm{P} 6$ and $\mathrm{P} 7$ peaks of $\mathrm{OH}$, and three fixed wavelength positions at the flank and top of the R-branch of the $\mathrm{O}_{2}$ band. The $2^{\circ}$ by $0.4^{\circ}$ field-of-view (corresponding to a rectangular area of $3 \mathrm{~km}$ by $0.6 \mathrm{~km}$ at the emission heights) has the longer side oriented east-west to minimize the effect of motions of the stellar background (for details, see Scheer, 1987; Scheer and Reisin, 1990).

Instrument bandwidth and peak transmission show much less degradation even at $30^{\circ}$ filter inclination angle (Scheer, 1987), than examples in the older literature. This feature seems to be essentially thanks to the small field of view and rectangular field aperture.

The intrinsic optical and mechanical robustness of this kind of instrument, together with the small dependence on gain variations characteristic of photon counting mode result in very stable photometric sensitivity. Therefore, the instrument does not require frequent recalibration. Although we do not make an absolute 


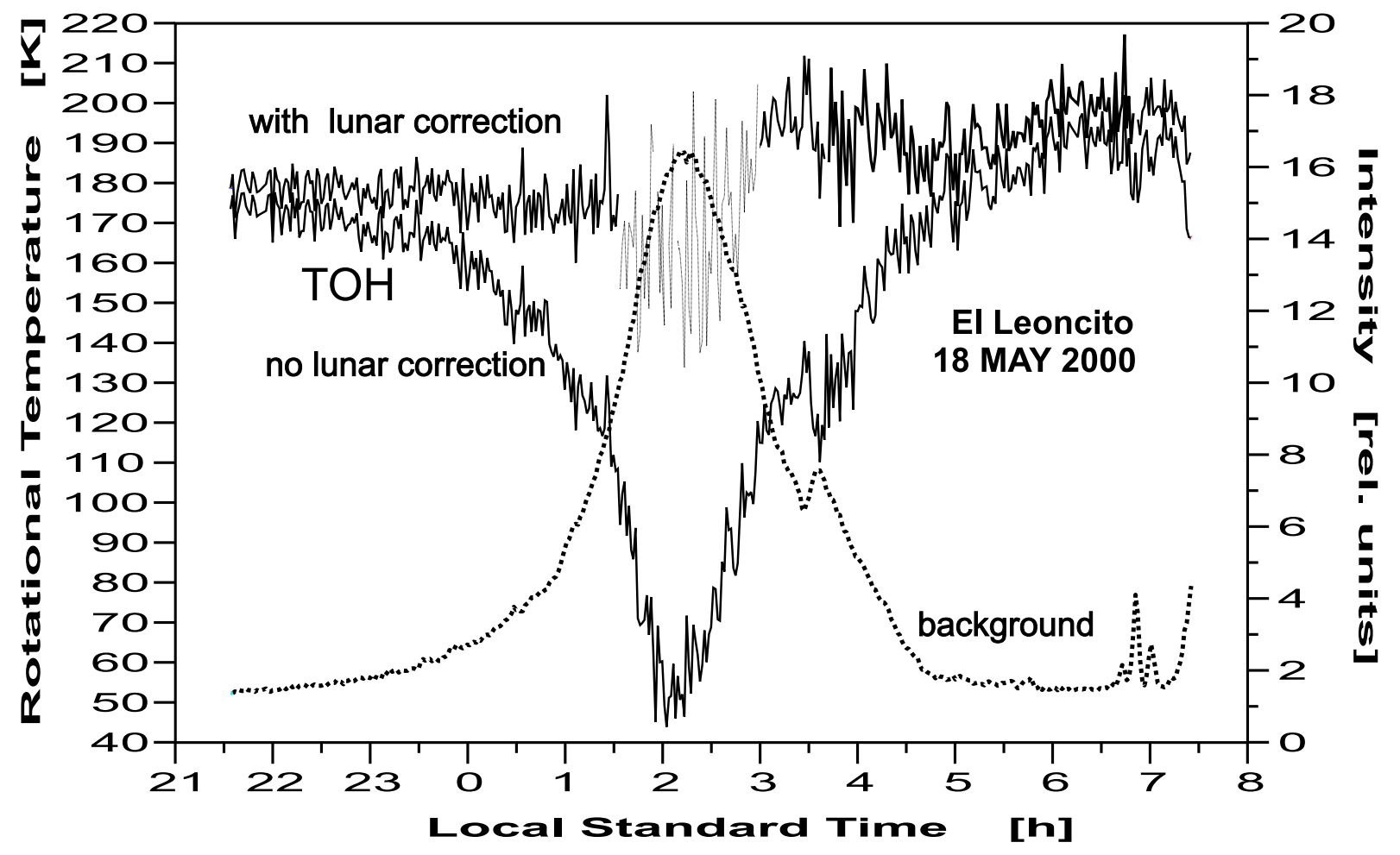

Fig. 1. Effect of lunar correction on $\mathrm{OH}$ temperatures on a full-moon night with strong atmospheric scattering. The solid lines belong to the uncorrected and corrected temperatures, and the dotted line to spectral background. The lighter shade corresponds to the unusable part of the data with lunar correction.

intensity calibration, the long-term constancy of the intensity scale has been verified by means of the stellar background (Scheer and Reisin, 2000).

Presently, each set of data is acquired in $81 \mathrm{~s}$, which includes the same total photon counting time of $67 \mathrm{~s}$ used since 1986 (thus, the difference due to stepping motor and computational overhead is now rather small). The main reason for the high effective measuring speed is the discrete sampling of a small number of spectral positions. The positions are sampled twice, sequentially in both spectral scan directions, to obtain mean values less biassed by airglow variations (by eliminating, to first order, spurious correlations between temperature and the first time derivative of intensity). This also leads to the intensity and temperature data of both emissions having the same temporal center point, as mentioned.

Rotational temperatures are determined by a combination of synthetic spectra (for the optical characteristics of the instrument) and Shagaev's scheme as explained by Scheer (1987). More recently, we have also incorporated the calculation of integrated band intensities (important for extracting dynamical information) from the spectral samples using rotational temperatures and synthetic spectra.

\section{Background corrections}

The raw intensity samples must be corrected for spectral background in order to avoid variable systematic errors in the derived intensities and temperatures. This is particularly important for avoiding spurious correlations between both parameters that would impair conclusions drawn from phase differences, especially in the context of gravity wave analysis (e.g., Reisin and Scheer, 2000). Corrections suitable for each sampling position can be derived rather straightforwardly from the background sample, plausible assumptions about the spectral shape, and the known instrument spectral response. For our sampling positions, the assumption of constant background already results in a very good supression of star signatures in the temperature and intensity data.

The background created by moonlight can be corrected in the same spirit, but by using the spectral shape determined empirically under very high background conditions. In this way, also Fraunhofer lines 


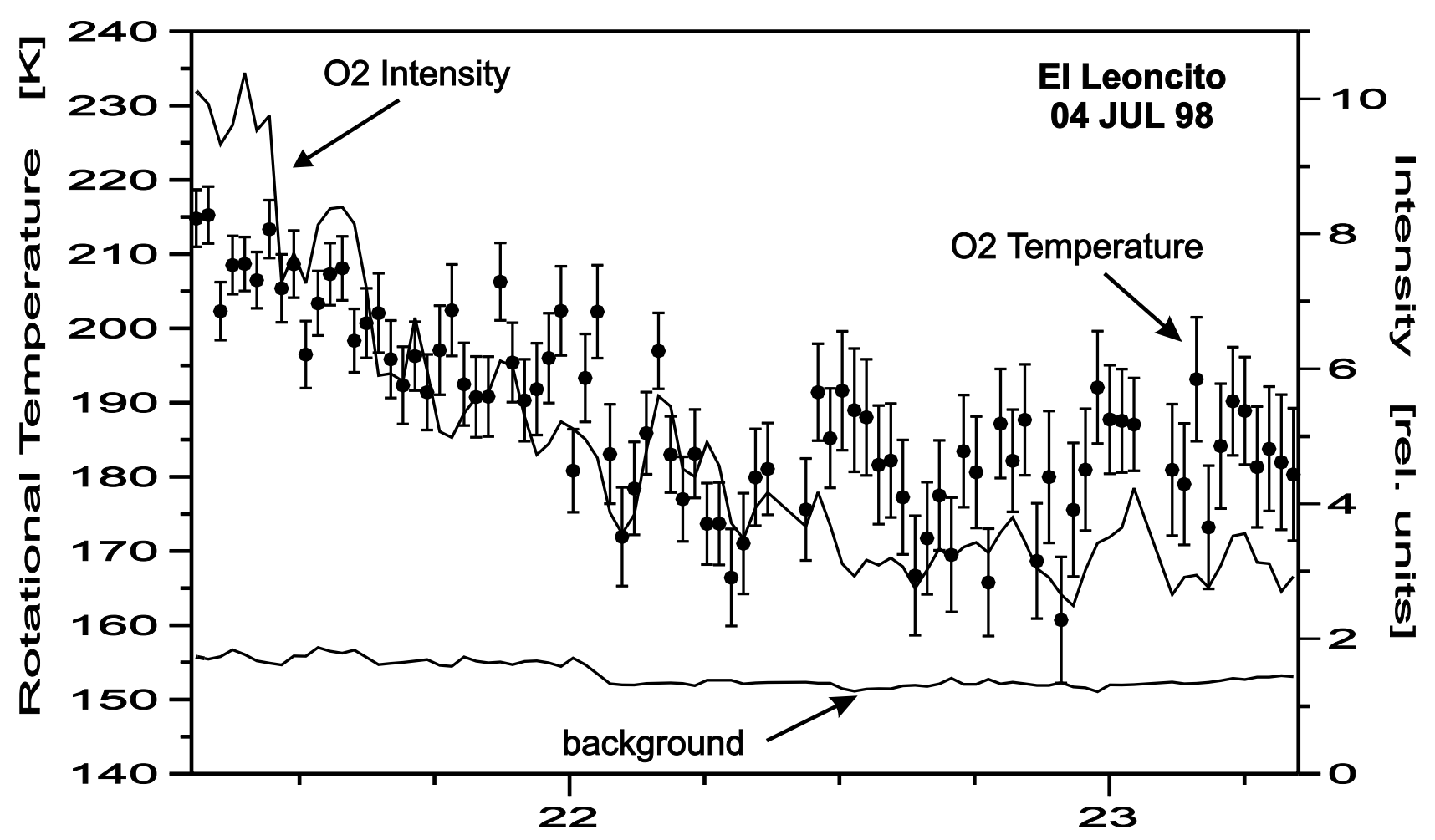

Local Standard Time [h]

Fig. 2. $\mathrm{O}_{2}$ temperatures (dots) including errors bars derived from photon statistics for a segment of the night July 4, 1998. $\mathrm{O}_{2}$ intensities and spectral background are shown as lines.

(in particular, the CaII line at about $854.2 \mathrm{~nm}$ that perturbs the $\mathrm{P} 6$ line of $\mathrm{OH}$ ), are automatically taken into account. The changeover between the two correction styles is done according to background level. The details of the correction can be tuned to optimize performance, under typical conditions, although it is impossible to obtain a correction valid under all scattering conditions.

As an example, the effect of the lunar correction on $\mathrm{OH}$ temperatures is shown in Figure 1, for a night with an extreme lunar background signal. This full moon night (18 May, 2000) had exceptionally strong atmospheric scattering. The $\mathrm{OH}$ temperatures with constant background correction are so much affected that they become very unrealistic, at closest lunar zenith approach $\left(14^{\circ}\right)$. In contrast, the lunar correction results in a nearly complete recovery of the data. However, we would normally reject about 3 hours of data, based on a criterion that compares background and airglow intensities. As shown in Figure 1, only about $1.5 \mathrm{~h}$ with maximum background (marked as a thin line) are really unusable, in this case, because the residues of the correction become non-negligeable and the noise becomes excessive. Our $\mathrm{O}_{2}$ temperatures (not shown) are much less sensitive to moonlight because of the closer spacing of samples and the absence of nearby Fraunhofer lines.

Backgrounds from other sources like city lighting, important during our Buenos Aires campaigns in 1988 and 1994, have been corrected in a similar fashion.

\section{Statistical errors}

Statistical errors are essentially due to photon statistics, and therefore can be derived with good accuracy from the set of sample counts, for each individual temperature and band intensity value. They depend on the (highly variable) airglow intensities, the spectral background, and photomultiplier dark count. Their precise knowledge is also useful input for realistic simulations of the complete data acquisition procedure. Figure 2 shows as an example a 2 hour section of a night with strong airglow brightness variation, and relatively high background. $\mathrm{O}_{2}$ temperatures are plotted as points with their associated error bars. The 
figure illustrates clearly how errors grow with decreasing band intensity, and how they still compete with the geophysical variability. The lower error values until about 21:30 LT are close to normal. Under optimum (maximum intensity) conditions, the temperature error can be as low as $\pm 2.5 \mathrm{~K}$, for $\mathrm{O}_{2}$, and $\pm 2.0 \mathrm{~K}$, for $\mathrm{OH}$. This error determination will enable us to derive a good estimate of the geophysical variability due to gravity waves.

\section{Data quality assurance}

Because of the high step resolution of the spectral scan mechanism (5000 steps over the spectral range), thermal drifts can be detected and compensated automatically with good precision, long before they can cause any noticeable effect on the data. Drifts are detected a few times per hour by scanning over a reference airglow peak (we now use the P5 peak of $\mathrm{OH}$ ). When the detection fails, the drift is calculated automatically from the change in filter chamber temperature. Sampling positions are then updated correspondingly, based on the wavelength calibrations determined over a wide range of ambient temperatures. The automatic corrections are later reviewed in a detailed analysis of the housekeeping data and the nocturnal correction history to detect any remaining positioning errors. The effect of theses errors on each sample intensity is then corrected, using the known spectral shape. This analysis also supplies a diagnostics of long-term mechanical changes that might eventually require repair.

Cloud absorption, on the one hand, affects the intensity data of both emissions and the background, simultaneously. On the other hand, geophysical intensity variations are not strongly correlated between both emissions. Therefore, short absorption episodes can be detected easily by comparing $\mathrm{OH}, \mathrm{O}_{2}$, and background intensities, and the data can be deleted, thus avoiding complete loss of (intensity) data in nights with variable cloud cover. Temperatures are not affected by moderate absorption, the only consequence being increased noise.

\section{Automatization}

The nocturnal data acquisition was already performed automatically, under the Radio Shack model 1 microcomputer control used in the past (Scheer 1987). However, the start and conclusion of the nightly observation run, and recovery from occasional faults required manual intervention, making unattended operation impossible. This was not a problem in the campaign style observations done until 1994.

Later, a special controller card for interfacing to a PC type computer was constructed, that implemented the same logic as the old interface (Scheer 1987). This change to PC control prepared the ground for obtaining more nearly continuous data, by removing the limitations of the old computer configuration. A very low-cost solution was found for prototyping the new interface that might be more generally useful. It consists in cementing the integrated circuits upside down, on both card faces, thus obtaining twice the component density possible with standard wire wrapping (but, of course, requiring a thorough layout and pinout documentation).

The PC control software was not completely redesigned, but adapted from the existing program that was written in the BASIC language, thus conserving the many useful and time-proven features of the original control philosophy (modularity, flexibility, fast upgrade capacity, manual intervention without data loss, etc.). The higher machine speed permits to replace the old Z80 machine code modules by BASIC routines. Obviously, the much more powerful PC performance results in higher reliability, faster data processing, and has permitted to add a great number of new features. It also has led directly to the improved instrument time resolution.

The change from an interrupt-less machine to a PC running under the MSDOS operating system called for the solution of some system problems to permit real time operation in time critical parts of the program, like software timing loops in the stepping motor control and temperature monitors. This requires, for example, that interrupts must temporarily be deactivated and timing loops calibrated against the real time clock to achieve reproducible software delays independent of hardware speed.

Presently, the complete operation cycle from daytime housekeeping to nighttime measurements has been automated, including recovery from many different fault conditions, which has made is possible to operate the instrument without human intervention. 


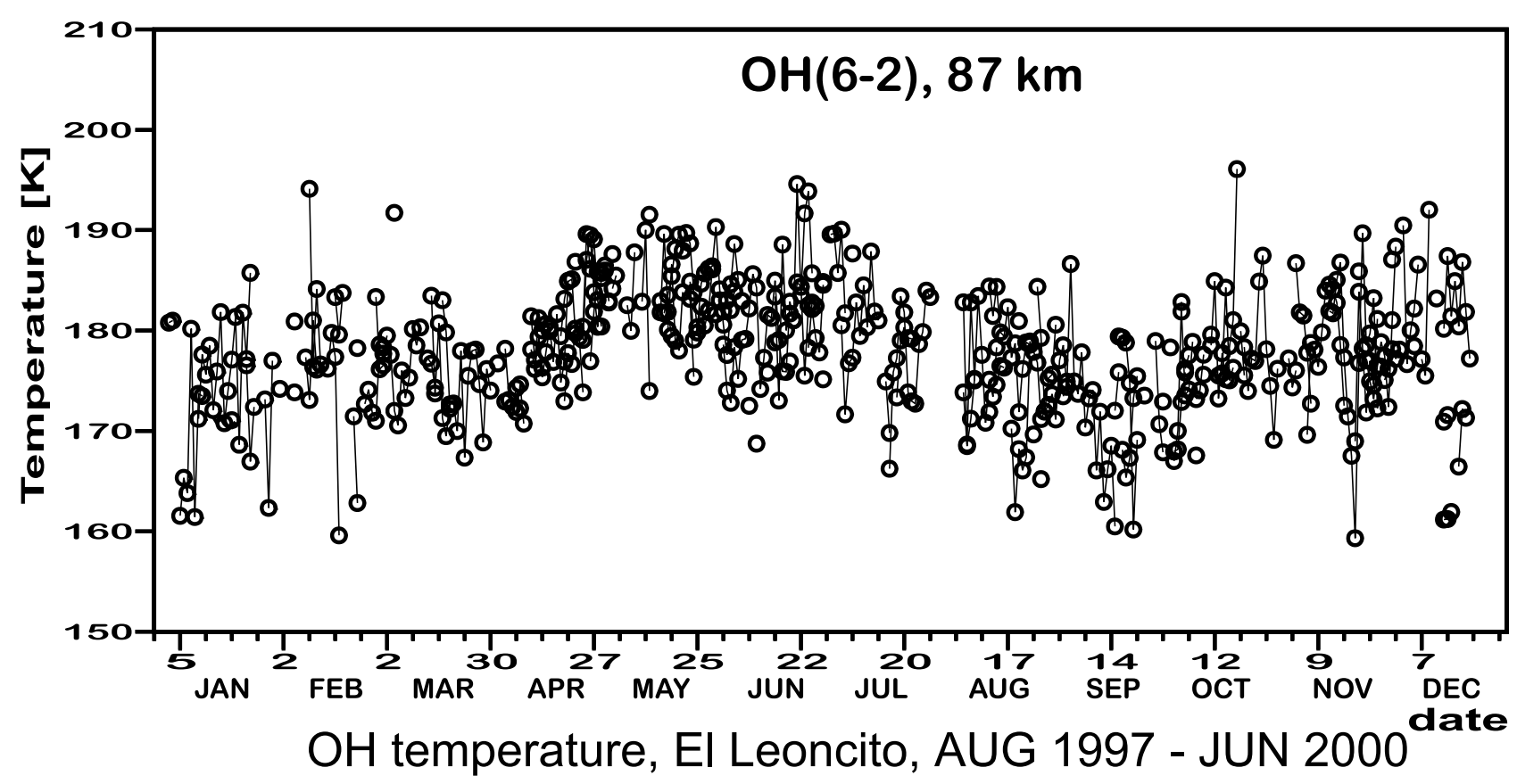

Fig. 3. Nocturnal means of $\mathrm{OH}$ temperatures at El Leoncito, from 5 August 1997 to 28 June 2000. Points for neighboring nights are connected by straight lines.

\section{SOME RECENT RESULTS}

Motivated by the desire to take part in international observation programs like the Planetary Scale Mesopause Observing System (PSMOS), a new series of observations from El Leoncito $\left(32^{\circ} \mathrm{S}, 69^{\circ} \mathrm{W}\right)$ was started in August 1997, that has led to quasi-continuous operation over the following years. With an annual data coverage of about 200 nights, and several months with more than 24 nights of observation, the seasonal variations have now been documented quite completely, until the present moment (28 June 2000).

As an example, Figure 3 shows the nocturnal means of $\mathrm{OH}$ rotational temperature for this new data set (about 470 points), versus the annual cycle (statistical error bars are not shown, because they do not generally exceed the size of the individual circles; the systematical error might be $10 \mathrm{~K}$ or larger, but this is irrelevant here). All the years are shown in black, because there is little interannual variability, on the average. The highest temperatures occur between late April and the end of June, and the minimum is reached around the spring equinox. This is completely unusual, since the traditional view consistent with many observations (mostly from the Northern Hemisphere, e.g. She and Lowe, 1998) expects high winter and low summer temperatures essentially centered on solstices. The details of this variation over the recent years, and of the other three parameters, will be treated in a forthcoming paper.

The seasonal behaviour of intensities, also including our older data, has been shown in Scheer and Reisin (2000). This paper focusses on the extremely low intensities in 1997.

The noise level and time resolution are adequate also for studying fast variations like gravity waves. It is possible to derive information on vertical wave propagation from the relation between intensity and temperature variations of each airglow emission, independently (Hines and Tarasick, 1987; Reisin and Scheer, 1996). For zenith observations, this requires neither winds nor horizontal wave characteristics. Our recent results signal the presence of a considerable proportion of downward wave propagation (Reisin and Scheer, 2000). This proportion is greater in the lower (OH) emission layer.

\section{CONCLUSIONS}

The simultaneous coverage of two airglow emissions from different altitudes increases the amount of useful geophysical information obtained from a single instrument.

Corrections for Fraunhofer absorptions in the solar spectrum during high background conditions ensure 
data quality even for measurements taken at full moon. This is also efficiently increases monthly data coverage.

Statistical errors can be derived a-priori from photon statistics, which is useful for a quantitative analysis of data variance and gravity wave activity.

Nearly completely automatic operation by PC control has also been a factor in achieving data coverage of about 200 nights per year, over the recent years.

The principal disadvantage of zenith observations like ours is that it is not possible to determine intrinsic wave periods. This would require not only information on horizontal wave propagation, but also wind information. These disadvantages can be remedied by combined instrumentation as, for example, reported by Takahashi et al. (1998).

\section{ACKNOWLEDGEMENTS}

The authors gratefully acknowledge the active support by the staff of Complejo Astronómico El Leoncito. This personal care and feeding of "El Bicho" can never be supplanted by any automatism. This work was partially funded by CONICET and ANPCyT grants PMP PICT 0137 and 07-00000-01818 / PICT '97-1818.

\section{REFERENCES}

Hines C. O., and D. W. Tarasick, On the Detection and Utilization of Gravity Waves in Airglow Studies, Planet. Space Sci. 35, 851-866, 1987.

Reisin, E. R., and J. Scheer, Characteristics of Atmospheric Waves in the Tidal Period Range Derived from Zenith Observations of $\mathrm{O}_{2} b(0-1)$ Atmospheric and $\mathrm{OH}(6-2)$ Airglow at Lower Mid-latitudes, J. Geophys. Res. 101, 21223-21232, 1996.

Reisin, E. R., and J. Scheer, Vertical Propagation of Gravity Waves Determined from Zenith Observations of Airglow, Adv. Space Res., submitted, 2000.

Scheer, J., Programmable Tilting Filter Spectrometer for Studying Gravity Waves in the Upper Atmosphere, Appl. Opt. 26, 3077-3082, 1987.

Scheer, J. and E. R. Reisin, Rotational Temperatures for $\mathrm{OH}$ and $\mathrm{O}_{2}$ Airglow Bands Measured Simultaneously from El Leoncito (314ㅇ'S), J. Atmos. Terr. Phys. 52, 47-57, 1990.

Scheer, J., and E. R. Reisin, Unusually Low Airglow Intensities in the Southern Hemisphere Midlatitude Mesopause Region, Earth Planets and Space 52, 261-266, 2000.

She, C.Y., and R. P. Lowe, Seasonal Temperature Variations in the Mesopause Region at Mid-Latitude: Comparison of Lidar and Hydroxyl Rotational Temperatures using WINDII/UARS OH Height Profiles, J. Atmos. Solar-Terr. Phys. 60, 1573-1583, 1998.

Takahashi, H., P. P. Batista, R. A. Buriti, D. Gobbi, T. Nakamura, T. Tsuda, and S. Fukao, Simultaneous Measurements of the Airglow $\mathrm{OH}$ Emission and Meteor Wind by a Scanning Photometer and the MU Radar, J. Atmos. Solar-Terr. Phys. 60, 1649-1668, 1998.

Taylor, M. J., W. R. Pendleton Jr., C. S. Gardner, and R. J. States, Comparison of Terdiurnal Tidal Oscillations in Mesospheric $\mathrm{OH}$ Rotational Temperature and Na Lidar Temperature Measurements at mid-Latitudes for Fall/Spring Conditions, Earth Planets and Space 51, 877-885, 1999.

published in Advances in Space Research, 27(6-7)1153-1158 (2001) 